\title{
THE ORBITAL STABILITY OF THE SUN-JUPITER-SATURN
}

\author{
SYSTEM
}

INVESTIGATION OF THE TRIPLE SYSTEM BY MASS ENHANCEMENT AND VARIATION OF ECCENTRICITY

\author{
J. R. DONNISON AND D. F. MIKULSKIS \\ Department of Mathematical Sciences \\ Goldsmiths College, University of London \\ New Cross, London, SE14 $6 \mathrm{NW}$
}

\begin{abstract}
Kuiper(1973) suggested that the stability of the Solar System may be meaningfully investigated by studying the stability of the SunJupiter-Saturn system. Numerical investigations by Nacozy(1976) showed that mass enhancement of the two planets beyond a factor of 29.25 led to instabilities in the system. In this new investigation similar mass enhancements were studied in detail numerically and compared with the analytical values derived from the $c^{2} H$ method. In addition, the eccentricities of the two planets were varied as well as their masses. It was found that the system soon showed signs of instability for the increased eccentricities when the masses of the planets were enhanced by fairly small factors.
\end{abstract}

\section{Introduction}

Complex systems such as star clusters can be broken down into a series of hierarchical systems where successive separations of the component masses increase by large factors and can be described by a series of two-body motions. In particular,hierarchical three-body systems composed of a binary system with a third body moving on an approximately Keplerian orbit relative to the centre of mass of the binary has been investigated in some detail by number of authors (see Donnison \& Mikulskis (1995)). It was suggested by Kuiper(1973) that the stability of such triple systems might have a strong bearing on the stability of the solar system, with the stability of the Sun-Jupiter-Saturn system being the dominant criterion for the continued existence of the planetary system. The stability of this system was investigated analytically by Szebehely and McKenzie (1977), and by 
Nacozy(1976) by short-time numerical integration of the system by increasing the masses of Jupiter and Saturn. This enhancement of masses allows any instabilities to emerge more rapidly and thus evolve the system more quickly.

For comparison, a restricted three-body Hill model with the mass of Saturn taken to be infinitesimal and both the planetary orbits assumed to be circular gives a critical planetary mass enhancement factor $\gamma$ for Jupiter of 18.45. A full three-body planar model using the $c^{2} H$ parameter ( $c$ angular momentum and $H$ the energy of the system) to control the topology of the zero-velocity surfaces, gave, using a two-body approximation for the energy, a value of 13.65 (Szebehely and McKenzie (1977). An improved model by Walker and Roy (1981) with the exact expression for the energy and a range of initial configurations reflected by a range of true anomalies gave $18.34 \leq$ $\gamma \leq 24.16$, where the upper limit represents a straight-line configuration with Jupiter at aphelion and Saturn at perihelion. These models maintain hierarchical or Hill stability with exchange of the component masses or crossing of the orbits ruled out, though the escape of Saturn from the system would not violate such criteria. To establish more general orbital stability, Nacozy(1976) integrated the system numerically for a wide range of $\gamma$. No secular trends were found for $\gamma \leq 29.0$, though as $\gamma$ was increased beyond $\gamma=29.25$ such trends became immediately apparent in all the orbital elements, with Saturn eventually being ejected in a few thousand years. In the current investigation the numerical approach was extended to cover variations in the eccentricities of both Jupiter and Saturn as well as $\gamma$.

\section{Dynamical model}

In Jacobian coordinates the equations governing the motion are

$$
\underline{\underline{r}}_{J}=-G\left(M_{\odot}+M_{J}\right)\left[\frac{\underline{r}_{J}}{r_{J}^{3}}+\frac{M_{S}}{M_{\odot}+M_{J}}\left(\frac{\underline{r}_{S}}{r_{S}^{3}}-\frac{\underline{r}_{J S}}{r_{J S}^{3}}\right)\right]
$$

and

$$
\underline{\ddot{r}}_{C S}=-G \frac{M_{\odot}+M_{J}+M_{S}}{M_{\odot}+M_{J}}\left(\frac{M_{\odot}}{r_{S}^{3}} \underline{r}_{S}+\frac{M_{J}}{r_{J S}^{3}} \underline{r}_{J S}\right)
$$

where $\underline{r}_{J}$ and $\underline{r}_{S}$ are the position vectors of Jupiter and Saturn with masses $M_{J}$ and $M_{S}$ relative to the Sun. $\underline{r}_{J S}=\underline{r}_{S}-\underline{r}_{J}$ and $\underline{r}_{C S}$ is the position of the vector of Saturn relative to the barycentre of the Sun-Jupiter system.

These equations were integrated numerically using a 12(10) embedded Runge-Kutta-Nystrom procedure (Dormand et al(1987)), with variable step length adjusted to a tolerance of $10^{-14}$. The mass, position and velocity of 
each component mass was specified for a straight line configuration simulating maximum perturbations. The stability criterion was that of Laplace where the orbital elements show no secular or large periodic variations over the time scale of the integration, in practice this meant that instability was deemed to have set in if the semi-major axis increased by $10 \%$ or the eccentricity by 0.1 . An additional check on the performance of the numerical code was the maintenance to high degree of accuracy of the constancy of the energy integral. The various systems considered were evolved numerically for about 1000 orbits of the Sun-Jupiter subsystem and in some cases for substantially more. In most cases any instability present tended to become apparent within the first 100 orbits and was marked by rapid secular changes in the orbital elements.

\section{Results}

In the first set of experiments the system was integrated for a range of $\gamma$ between 20 and 100, with the orbital elements taken to have their present values. This is similar to the set of calculations to those carried out by Nacozy (1976). It was found that for $\gamma \leq 28$, the system obeyed our strict definition of stability. In the range $28.0 \leq \gamma \leq 29.2$, it was found that although the semi-major axes and eccentricities showed changes exceeding the $10 \%$ level, the changes appeared periodic in nature. For $\gamma>29.2$, the orbits were found to not only violate the basic criteria but were clearly secular, increasing rapidly with Saturn eventually escaping from the system altogether. These results agree with those of Nacozy(1976) who found clear indications of instability for $\gamma>29.25$; the value $\gamma=29.2$ was not integrated by Nacozy. It seems clear that Nacozy(1976) used a criteria relating to large changes in eccentricity to determine instability. Graziani and Black(1981), when testing their numerical code against Nacozy's results, found that the system was stable when $\gamma$ was 20 or less and strongly unstable when $\gamma$ of 30 or more was used. Nothing was explicitly stated about the intervening values.

In the next series of numerical experiments the eccentricity of Jupiter's orbit, $e_{J}$, was increased for the same range of $\gamma$ as before. Jupiter was thus initially released closer to Saturn producing greater perturbations in Saturn's orbit. The conditions under which varying the orbit of Jupiter will disrupt the orbit of Saturn and the nature of the disruption were determined. The results are displayed in table I. It was found that for an increase in $e_{J}$ to 0.1 only $\gamma=1$ was completely stable,though periodic variations were apparent for small changes in $e_{J}$ or small $\gamma$ values.These are likely to be unstable over longer time scales. The instabilities were largely, as expected,linked to the disturbance of Saturn's orbit which increases with 
$\gamma$ and $e_{J}$.

A similar series of numerical experiments were carried out by increasing the eccentricity of Saturn, $e_{S}$, while that of Jupiter remains at its current value. The initial perturbations on Saturn's orbit were thus increased compared to the present situation. The results are displayed in table II. The general mode of instability was the ejection of Saturn from the system with $e_{J}$ increased.

From the series of calculations performed it was clearly found that, as the mass ratio $\gamma$ was increased, instability in the present system became apparent as value around 29 were reached. Similar calculations varying $e_{J}$ or $e_{S}$, as well as $\gamma$, showed that instability sets in rapidly for all but the smallest changes in the eccentricities.

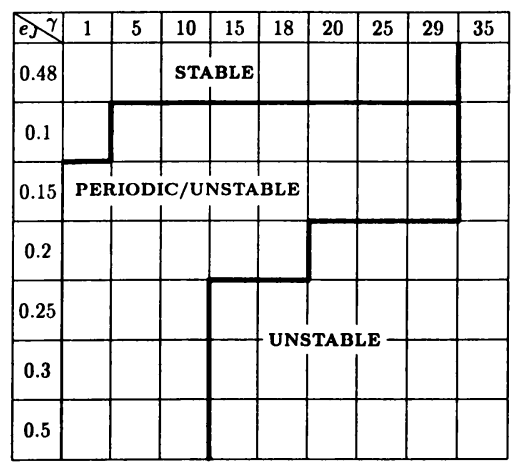

\begin{tabular}{|l|c|c|c|c|}
\hline \multicolumn{1}{|c|}{$\gamma$} & 15 & 18 & 20 & 35 \\
\hline 0.1 & STABLE & $\begin{array}{l}\text { PERIODIC } \\
\text { /STABLE }\end{array}$ & & \\
\hline 0.2 & $\begin{array}{l}\text { PERIODIC } \\
\text { /STABLE }\end{array}$ & & & \\
\hline 0.3 & $\begin{array}{l}\text { PERIODIC } \\
\text { /STABLE }\end{array}$ & & UNSTABLE & \\
\hline 0.5 & & & & \\
\hline 0.8 & & & & \\
\hline
\end{tabular}

Table I variations in $\gamma$ and $e_{J}$. Table II variations in $\gamma$ and $e_{S}$

\section{References}

Donnison J.R.\& Mikulskis, D.F.(1995) MNRAS, 272, 1.

Dormand J.R., El-Mikkawy M.E.A. \& Prince P.J.(1987) IMA J. Num. Analy., 7, 235.

Kuiper G.(1973) Celest. Mech.,9, 321.

Nacozy P.E.(1976) Astron. J.,81, 787.

Szebehely V.\& McKenzie R.(1977) Astron.J.,82, 79.

Walker I.W.\& Roy A.E.(1981) Celest. Mech.,24, 195. 